\title{
Disease management programs in heart failure: half a century of an unmet need
}

\author{
Deddo Moertl (iD
}

Received: 4 October 2017 / Accepted: 12 October 2017 / Published online: 14 November 2017 (C) The Author(s) 2017. This article is an open access publication.

inertia /ı'nə: $\int$ ə/: A tendency to do nothing or to remain unchanged. Oxford English Dictionary

Heart failure (HF) is a devastating disease with severe symptoms, reduced quality of life and a poor prognosis. Without appropriate treatment, HF has a higher mortality than many forms of cancer [1]. Hospitalization and readmission are common, which negatively affect patient quality of life and cause considerable health-economic costs [2].

Fortunately, there are good treatment options for chronic HF, in particular for patients with a reduced left ventricular ejection fraction. Disease-modifying therapies including angiotensin-converting enzyme (ACE) inhibitors, beta-adrenergic blockers, mineralocorticoid receptor blockers, cardiac resynchronization therapy and the recently marketed sacubitril/ valsartan showed positive effects on exercise capacity and quality of life as well as reducing hospitalization and mortality rates [3]. Just with appropriate drug treatment we can triple the patients' remaining life span [4].

Despite these overwhelming benefits shown in clinical trials, guideline-recommended treatment is not delivered to the real-world patient. Data from 13 Austrian health insurance funds analyzing $>36,000$ patients showed a disastrous drug adherence after discharge from HF hospitalization. After a median of 614 days, prescriptions for ACE inhibitors, beta-adrener-

\section{Moertl, MD, FHFA ( $₫)$}

Clinical Department of Internal Medicine III, University Hospital St. Poelten, Karl Landsteiner University of Health Sciences, Propst Fuehrer-Straße 4, 3100 St. Poelten, Austria deddo.moertl@meduniwien.ac.at

Karl Landsteiner Institute for the Research of Ischemic Cardiac Diseases and Rhythmology, St. Poelten, Austria gic blockers, and mineralocorticoid receptor-antagonists were filled in only $49.3 \%, 40.4 \%$, and $16.1 \%$ of the cases, respectively [5]. Thus, the question arises how implementation of HF guidelines can be improved. One option is the inclusion of HF patients in a multidisciplinary disease management program (DMP), which has received a class I recommendation in international HF guidelines for more than 10 years [3, 6]. Such programs can improve patient well-being, reduce hospitalizations and prevent premature death [7]. Beyond these clinical benefits, these programs have proven to be cost-effective and some even costsaving [8]; however, apart from local initiatives with a high variety in design, duration, size and effect, no nationwide structured HF service has so far been implemented in Austria. Therefore, the Heart Failure Working Group of the Austrian Society of Cardiology has decided to elaborate a position paper on DMPs for chronic HF within the Austrian context. This position paper aims to provide evidence-based arguments for the need and efficacy of a comprehensive DMP for HF and to describe the essential components of such a program. It is based on the recommendations of the European Society of Cardiology (ESC) [9], which were adapted to Austrian circumstances. The key elements are multidisciplinary treatment including physician and nurse HF specialists, a seamless integration of all sectors of care from primary care to tertiary centers, HF outpatient clinics serving as expert referral centers for the entire network, and adherence to guidelines. Integrated HF management within a DMP needs to be coordinated, which in most models is done by a specialist HF nurse. Other tasks of this nurse can be implementation of home visits, supportive monitoring of treatment optimization, early recognition of worsening HF, and facilitation of patient empowerment.

While this position paper has been urgently needed and its importance for the promotion of guideline- 
recommended treatment in Austria is obvious, it must be noted that the demands made are not new. For example, in an article published in the American Journal of Public Health in the 1960s entitled "Congestive heart failure, the patient and the community", Raymond T. Benack already outlined a DMP for chronic HF [10]. Accounting for the huge number of patients, their complexity and poor prognosis he recommended a multidisciplinary approach. Nurses trained in HF should perform home visits, check physical status and adherence to recommendations, and report to the treating physician. For Benack the main benefit of home-based nursing care for HF was a reduction in hospitalization, mainly achieved by early detection of worsening HF. Thus, he concluded "Through these combined services the patient will receive better home care, recurrences of congestive heart failure can be controlled, and the community and the patient will show a financial saving through decreased hospital readmissions and decreased total hospital time. Finally, of course, the patient will enjoy a longer and more useful life because of the prevention of complications from repeated attacks of congestive heart failure."

Despite being published in 1964, the basic characteristics of a DMP for HF as described by Benack are still valid today and in the main do not differ from the positions presented in the Austrian paper [11] or the corresponding ESC statement [9]. While the cardiac community is often considered to be a fast adopter of innovations, at least when we think of interventional technologies, the lack of DMPs seems even more striking considering that Benack's paper was published more than half a century ago and randomized controlled trials demonstrating the benefits of DMPs date back to the 1990s [12]. Obviously, there is some ongoing inertia of the responsible healthcare authorities to implement this class I A recommendation for decades, and the reasons for this impressive example of nonimplementation of evidence-based medicine are definitely complex and hard to understand.

Where do we go from here? The position paper makes a strong claim for the nationwide implementation of structured HF management; however, is it necessary to implement a one-size-fits-all program for all regions? Probably not. Some diversity in design of DMPs for HF will be acceptable, provided certain characteristics and components are implemented and the clinical benefit and the cost-effectiveness is guaranteed and regularly scrutinized. The next step should be the evaluation of ongoing DMPs according to the requirements given in the position paper. Quality control and regular audits as integrated parts of a DMP will be essential items for evaluation, giving insights into efficacy and cost-effectiveness of specific DMPs.

Regions without DMPs for HF should implement one as soon as possible and the presented position paper could serve as a blueprint; however, implementation should not rely on the ambitious initiatives of local opinion leaders trying to raise funds via various sources. Since the evidence for the clinical and economic benefit is clear and given a class IA recommendation in the European guidelines, it is the urgent responsibility of the official healthcare authorities to establish and run DMPs for HF.

Conflict of interests D. Moertl declares that he has no competing interests.

Open Access This article is distributed under the terms of the Creative Commons Attribution 4.0 International License (http://creativecommons.org/licenses/by/4.0/), which permits unrestricted use, distribution, and reproduction in any medium, provided you give appropriate credit to the original author(s) and the source, provide a link to the Creative Commons license, and indicate if changes were made.

\section{References}

1. Stewart S, MacIntyre K, Hole DJ, Capewell S, McMurray JJ. More 'malignant' than cancer? Five-year survival following a first admission for heart failure. Eur J Heart Fail. 2001;3(3):315-22.

2. Stewart S, Jenkins A, Buchan S, McGuire A, Capewell S, McMurrayJJ.The current cost of heartfailure to the National Health Service in the UK. Eur JHeartFail. 2002;4(3):361-71.

3. Ponikowski P, Voors AA, Anker SD, Bueno H, Cleland JG, Coats AJ, et al. 2016 ESC Guidelines for the diagnosis and treatment of acute and chronic heart failure: The Task Force for the diagnosis and treatment of acute and chronic heart failure of the European Society of Cardiology (ESC)Developed with the special contribution of the Heart Failure Association (HFA) of the ESC. Eur Heart J. 2016;37(27):2129-200.

4. Cole GD, Patel SJ, Zaman N, Barron AJ, Raphael CE, Mayet J, etal. "Triple therapy" of heart failure with angiotensin-converting enzyme inhibitor, beta-blocker, and aldosterone antagonist may triple survival time: shouldn't we tell patients? JACC Heart Fail. 2014;2(5):545-8.

5. Marzluf BA, Reichardt B, Neuhofer LM, Kogler B, Wolzt M. Influence of drug adherence and medical care on heart failure outcome in the primary care setting in Austria. PharmacoepidemiolDrug Saf. 2015;24(7):722-30.

6. Swedberg K, Cleland J, Dargie H, Drexler H, Follath F, Komajda M, et al. Guidelines for the diagnosis and treatment of chronic heart failure: executive summary (update 2005): The Task Force for the Diagnosis and Treatment of Chronic Heart Failure of the European Society of Cardiology. Eur HeartJ. 2005;26(11):1115-40.

7. Takeda A, Taylor SJ, Taylor RS, Khan F, Krum H, Underwood M. Clinical service organisation for heart failure. Cochrane Database Syst Rev. 2012; https://doi.org/10. 1002/14651858.CD002752.pub3.

8. MoertlD, Steiner S, CoyleD, Berger R. Cost-utility analysis of nt-probnp-guided multidisciplinary care in chronic heart failure. IntJ Technol Assess Health Care. 2013;29(1):3-11.

9. McDonagh TA, Blue L, Clark AL, Dahlstrom U, Ekman I, Lainscak M, et al. European society of cardiology heart failure association standards for delivering heart failure care. Eur J Heart Fail. 2011;13(3):235-41.

10. Benack RT. Congestive heart failure, the patient and the community. Am J Public Health. 1964;54(10):4. 
11. MoertlD, Altenberger J, Bauer N et al. Disease management programs in chronicheart failure: Position statement of the Heart Failure Working Group and the Working Group of the Cardiological Assistance and Care Personnel of the Austrian Society ofCardiology. Wien Klin Wochenschr. 2017. https:// doi.org/doi.org/10.1007/s00508-017-1265-0.
12. West JA, Miller NH, Parker KM, Senneca D, Ghandour G, Clark M, et al. A comprehensive management system for heart failure improves clinical outcomes and reduces medical resource utilization. Am J Cardiol. 1997;79(1):58-63. 